\title{
Exceptional Therapeutic Outcome of Metastatic Neuroendocrine Tumor with Peptide Receptor Radionuclide Therapy with Brief Review of Literature
}

\author{
Manoj Gupta ${ }^{1^{*}}$, Partha S Choudhury ${ }^{1}$, Shivendra Singh ${ }^{2}$ and Anurag Mehta ${ }^{3}$ \\ ${ }^{1}$ Department of Nuclear Medicine, Rajiv Gandhi Cancer Institute and Research Centre, Sector 5, Rohini, New Delhi, India \\ ${ }^{2}$ Department of GI-Oncosurgery, Rajiv Gandhi Cancer Institute and Research Centre, Sector 5, Rohini, New Delhi, India \\ ${ }^{3}$ Department of Pathology, Rajiv Gandhi Cancer Institute and Research Centre, Sector 5, Rohini, New Delhi, India
}

*Corresponding author: Manoj Gupta, Department of Nuclear Medicine, Rajiv Gandhi Cancer Institute and Research Centre, Sector 5, Rohini, New Delhi, India, Tel: 919540907949; Fax: 911127051037; E-mail: docmanojgupta@yahoo.com

Received date: May 17, 2017; Accepted date: May 25, 2017; Published date: May 30, 2017

Copyright: ( 2017 Gupta M, et al. This is an open-access article distributed under the terms of the Creative Commons Attribution License, which permits unrestricted use, distribution, and reproduction in any medium, provided the original author and source are credited.

\begin{abstract}
NETs are rare, heterogeneous group of neoplasm presented as chronic oncologic disease. Somatostatin analogue is the standard first line systemic therapy for mainly hormone control. No standard second line systemic treatment is available except everolimus which has no reported complete response. PRRT is an innovative molecular targeted treatment based on theragnostic concept for well differentiated NETs.
\end{abstract}

We presented here a 63-year-old lady with grade 1 NET of rectum with lymphnodal and liver metastasis. She underwent sigmoid colostomy for bowel symptoms and started on sandostatin LAR. After progression, patient was treated with 4 cycles of $7.4 \mathrm{GBq}$ of $177 \mathrm{Lu}$-DOTATATE at 10 weeks interval. No hematological and renal toxicity were noticed. Patient showed complete response in liver lesions \& lymphnodes and partial response in rectal lesion on 68Ga-DOTANOC PET-CT. After multispecialty clinic board discussion, patient underwent curative surgery for residual rectal lesion and colostomy closer later on.

Our case highlights a common presentation of NETs but an uncommon outcome with currently approved drugs. With this potential of disease cure in metastasis, PRRT may also be offered for locally advance disease as an adjuvant treatment for down staging.

Keywords: Neuroendocrine tumor; Peptide receptor radionuclide therapy; Theragnostic; 177Lu-DOTATATE; Complete response

\section{Introduction}

Neuroendocrine tumors (NETs) are relatively slow growing with $80 \%$ of patients presenting in stage IV and 5 year survival of $35 \%-55 \%$ [1]. Somatostatin analogue is the standard first line systemic therapy for inoperable locally advanced or metastatic patient for both control of hormone secretion and tumor growth [2]. No standard second line systemic treatment option is available except everolimus. Peptide receptor radionuclide therapy (PRRT) is a promising targeted treatment for well differentiated (grade 1\&2) NETs using radiolabelled somatostatin analogue to deliver cytotoxic radiation at cellular level [3]. We presented here a successful treatment outcome of an inoperable metastatic NET case to disease free state following PRRT.

\section{Case Report}

A 63-year-old lady was presented with difficult defecation and occasional red coloured stool since 2 years. Triple phase contrast enhanced computed tomography whole abdomen (TP-CECT WA) showed upper 1/3rd rectal lesion with perirectal, paraaortic lymphadenopathy and liver lesions. Colonoscopy revealed rectal growth and biopsy of which showed grade 1 (Ki-67<2\%) NET. 68Gallium-DOTA1-NaI3-octreotide (68Ga-DOTANOC) Positron Emission Tomography- Computed tomography (PET-CT) showed strong (more than spleen) somatostatin receptor expression in all sites.
Initial Chromogranin A (CgA) and 5-hydroxyindoleacetic acid (5HIAA) were $312.2 \mathrm{ng} / \mathrm{ml}$ and $5.4 \mathrm{ng} / 24$ hours urine respectively. Sigmoid loop colostomy was done due to inoperable rectal lesion and she was started on sandostatin LAR (long acting release) $30 \mathrm{mg} 4$ weekly. 5 months later, she was re-evaluated with 68Ga-DOTANOC PET-CT with TP-CECT WA which revealed progressive disease. Case was discussed in multispecialty clinic (MSC) board and PRRT was advised. She was treated with 4 cycles of $200 \mathrm{mCi}$ (7.4GBq) 177Lutetium-DOTA0-Tyr3-octretate (177Lu-DOTATATE) at 10 weeks interval with renal protection protocol (2 litres Aminoven $10 \% 500 \mathrm{ml}$ at 1 hour $+500 \mathrm{ml}$ Gelofusine $100 \mathrm{ml} /$ one hour) started 30 minutes before radiopharmaceutical infusion. Biochemical tests at 1,4 , and 8 weeks intervals after each cycle for complete blood count, kidney function test and liver function test were done which showed no toxicity. $\mathrm{CgA}$ at 8 weeks interval of each cycle showed decreasing trends $(354.2 \mathrm{ng} / \mathrm{ml}$ at base line and $105.9 \mathrm{ng} / \mathrm{ml}$ after 4 cycles of PRRT). 99mTc-DTPA glomerular filtration rate (GFR) at 8 weeks interval of each cycle showed no derangement $(109.6 \mathrm{ml} / \mathrm{min}$ GFR at base line and $101.5 \mathrm{ml} / \mathrm{ml}$ after 4 cycles of PRRT). 68Ga-DOTANOC PET-CT with TP-CECT WA scan was done at 8 weeks interval for response evaluation which showed serially decrease in tracer intensity and size in all disease sites (Figure 1). Lymphnodes and liver lesions showed complete response (CR) after 4 cycles of PRRT. Residual rectal lesion after 4 cycles of PRRT was surgically removed (Lower anterior resection with side to end colorectal anastomosis with covering transverse loop colostomy) after MSC board decision. Histopathology was residual rectal NET grade 1 (Figure 2) with $1 / 6$ perirectal 
Citation: Gupta M, Choudhury PS, Singh S, Mehta A (2017) Exceptional Therapeutic Outcome of Metastatic Neuroendocrine Tumor with Peptide Receptor Radionuclide Therapy with Brief Review of Literature. J Nucl Med Radiat Ther 8: 333. doi:10.4172/2155-9619.1000333

Page 2 of 3

lymphnodes positive (ypT3N1). 6 months post-surgery $68 \mathrm{Ga}-\quad$ normal. CgA was $52.5 \mathrm{ng} / \mathrm{ml}$. Colostomy reversal done and patient was DOTANOC PET-CT with TP-CECT WA scan and colonoscopy were in disease free state since last 8 months of follow-up.

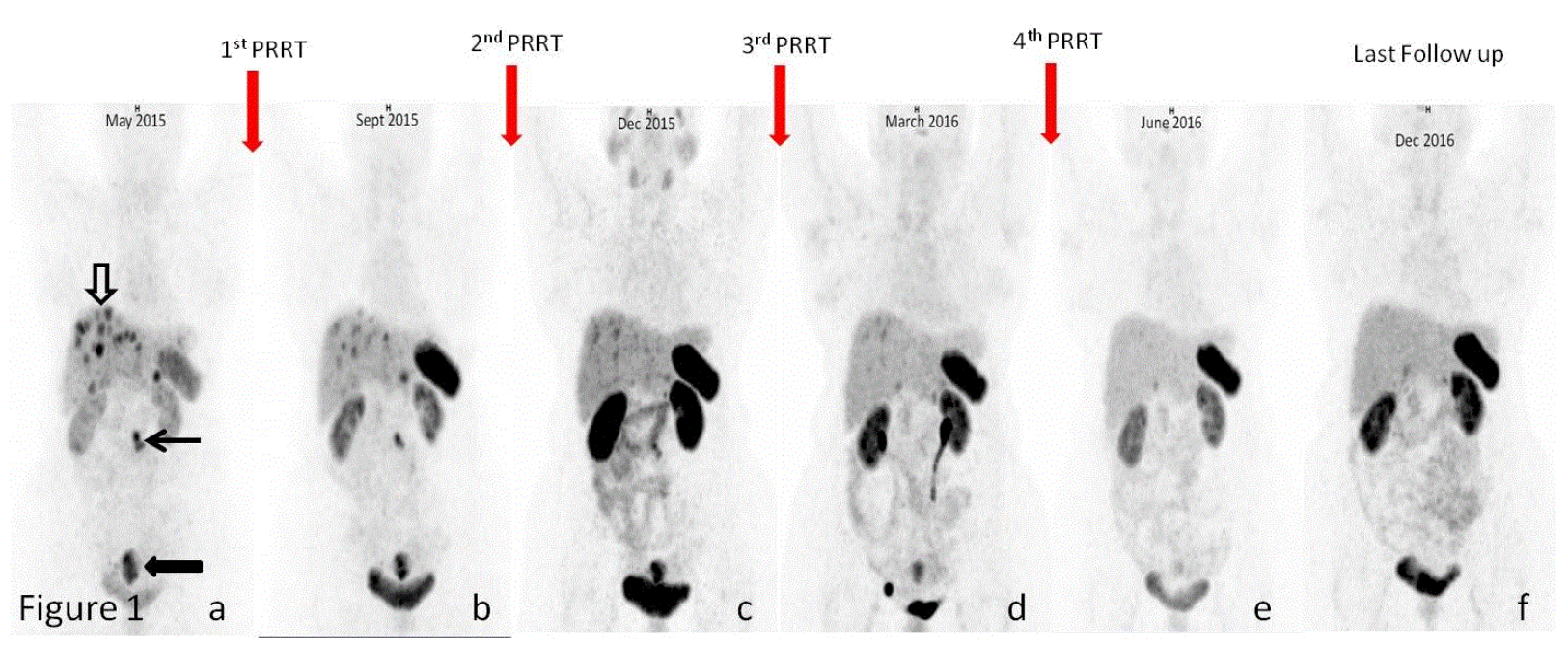

Figure 1: Serial 68Ga-DOTANOC PET-CT Maximum intensity projection images (a-f): Image (a) showed strong (more than spleen) somatostatin receptors (SSTRs) expressing rectal lesion (black block arrow), retroperitoneal lymphnodes (black arrow) and liver lesions (empty block arrow). Serial images (b to e) after each peptide receptor radionuclide therapy (PRRT) showed response in liver lesions and retroperitoneal lymphnodes. Last follow-up image (f) showed no abnormal SSTRs expressing lesion in the body.

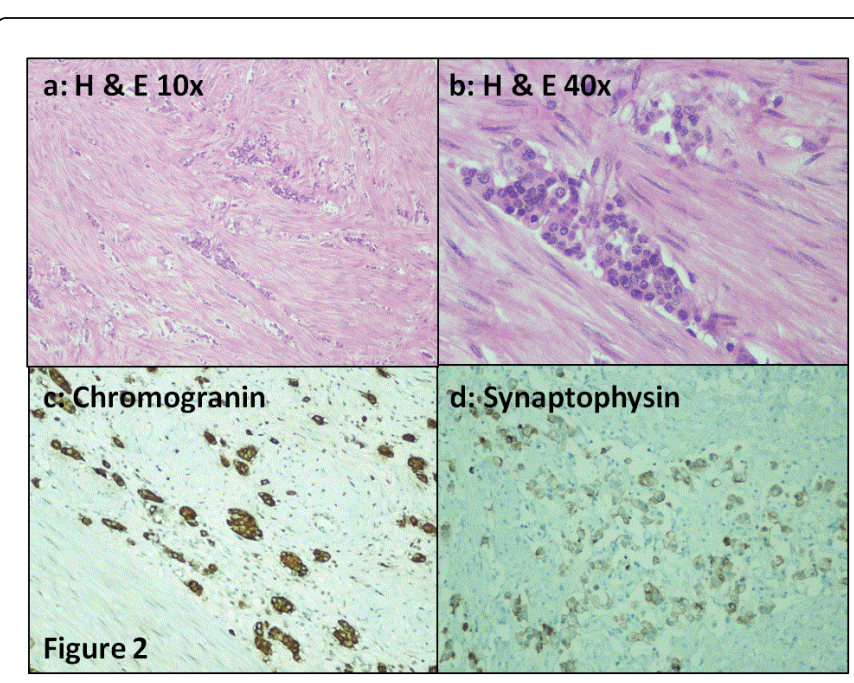

Figure 2: H\&E images (2a: 10X; 2b: 40X) shows rectal wall infiltrated by solid nests of tumor cells. IHC images show immunopositivity for chromogranin (2c) and synaptophysin (2d).

\section{Discussion}

NETs are rare, heterogeneous group of neoplasm presented as chronic oncologic disease. Incidence has increased over last two decades due to technical improvements in diagnosis with prevalence of 35 cases per 100,000 inhabitants [4]. However no systemic treatment is available with meaningful objective response rate for these patients. The first choice of treatment 'Sandostatin LAR' has median PFS of 14.3 months with $<3 \%$ partial response (PR) and no CR (PROMID study). In RADIANT-3 trial [5], everolimus an oral mammalian target of rapamycin (mTOR) inhibitor has showed median PFS of 11 months with $5 \%$ objective response rate (all PR).

PRRT is a molecular targeted treatment based on 'Theragnostic' concept [6]. In this concept, a specific antigen expression is investigated by an imaging agent and if present same antigen is being targeted by a therapeutic radiopharmaceutical. PRRT exploits the somatostatin receptors (SSTRs) over expression in grade 1 and 2 NETs (Ki67 index $\leq 20 \%$ ). 68Ga-DOTANOC PET-CT is used for imaging while $177 \mathrm{Lu}$-DOATATE for subsequent treatment. $68 \mathrm{Ga}$ is a positron emitting radionuclide with a half-life of 68 minutes and $177 \mathrm{Lu}$ is a $\beta$ and $\gamma$ emitting radionuclide with half-life of 6.7 days and $2 \mathrm{~mm}$ maximum particle range. In a single group study of gastroenteropancreatic NETs $(\mathrm{N}=310)$ treated with 177Lu-DOTATATE showed $2 \%$ CR, 28\% PR and median PFS of 33months [7]. A recent phase 3 trial (NETTER-1) of 177Lu-DOTATATE in mid gut NETs showed $18 \%$ objective response rate $(1 \% \mathrm{CR}+17 \% \mathrm{PR})$ with $65.2 \%$ median PFS at 20 months [8].

Our case highlights a common presentation of NETs but an uncommon outcome. More disturbing was sigmoid colostomy to our patient which was an inconvenience and restricting her social activities. After 4 cycles of PRRT she not only underwent curative surgery but colostomy reversal later on hence improved her quality of life.

\section{Conclusion}

We conclude NETs are rare but chronic oncological disease with rarest chance of cure in metastatic case as of now. PRRT is an innovative molecular targeted treatment with a potential of disease cure. It may also be offered for locally advance disease as an adjuvant treatment for down staging. With the growing literature on PRRT it may become one of the choice treatments in near future. 
Citation: Gupta M, Choudhury PS, Singh S, Mehta A (2017) Exceptional Therapeutic Outcome of Metastatic Neuroendocrine Tumor with Peptide Receptor Radionuclide Therapy with Brief Review of Literature. J Nucl Med Radiat Ther 8: 333. doi:10.4172/2155-9619.1000333

Page 3 of 3

\section{References}

1. Durante C, Boukheris H, Dromain C, Duvillard P, Leboulleux S, et al (2009) Prognostic factors influencing survival from metastatic (stage IV) gastroenteropancreatic well-differentiated endocrine carcinoma. Endocr Relat Cancer 16: 585-597.

2. Rinke A, Müller HH, Schade-Brittinger C, Klose KJ, Barth P, et al. (2009) Placebo-controlled, double-blind, prospective, randomized study on the effect of octreotide LAR in the control of tumor growth in patients with metastatic neuroendocrine midgut tumors: A report from the PROMID Study Group. J Clin Oncol 27: 4656-4663.

3. Van der Zwan WA, Bodei L, Mueller-Brand J, Wouter W de Herder, Larry $\mathrm{K}$ Kvols, et al. (2015) Radionuclide therapy in neuroendocrine tumors. European J Endocrinol 172: R1-R8.

4. Yao JC, Hassan M, Phan A, Dagohoy C, Leary C, et al. (2008) One hundred years after "carcinoid": Epidemiology of and prognostic factors for neuroendocrine tumors in 35,825 cases in the united states. J Clin Oncol 26: 3063-3072.

5. Yao JC, Shah MH, Ito T, Bohas CL, Wolin EM, et al. (2011) Everolimus for Advanced pancreatic neuroendocrine tumors. N Engl J Med 364: 514-523.

6. Oberg K (2012) Molecular imaging radiotherapy: Theranostics for personalized patient management of neuroendocrine tumors (NETs). Theranostics 2: 448-458.

7. Kwekkeboom DJ, de Herder WW, Kam BL, van Eijck CH, van Essen M, et al. (2008) Treatment with the radiolabeled somatostatin analog [177LuDOTA 0, Tyr3] octreotate: toxicity, efficacy, and survival. J Clin Oncol 26: 2124-2130.

8. Strosberg J, El-Haddad G, Wolin E, Hendifar A, Yao J, et al. (2017) Phase 3 trial of 177lu-dotatate for midgut neuroendocrine tumors. N Engl J Med $376: 125-135$ 